\title{
Residence Time Control of Systems Subject to Measurement Noise*
}

\author{
T. RUNOLFSSON ${ }^{\dagger}$ \\ Department of Electrical Engineering and Computer Science, \\ The University of Michigan, Ann Arbor, Michigan 48109-2122 \\ Submitted by S. M. Meerkov \\ Received December 15, 1987

\begin{abstract}
The residence time control problem is considered for linear systems that are subject to both input and measurement noise disturbances. It is shown that the maximal residence time is bounded and an upper bound is derived. Necessary and sufficient conditions for the existence of controllers that achieve the upper bound are derived and design techniques for residence time controllers are considered. Connections with optimal output feedback control are explored. The approach is based on an asymptotic version of the large deviations theory. 1990 Academic Press, Inc.
\end{abstract}

\section{INTRODUCTION}

Given a controlled dynamical system with states $x(t) \in \mathbb{R}^{n}$, control $u(t) \in \mathbb{R}^{m}$, output $y(t) \in \mathbb{R}^{p}$ and disturbances $\xi(t) \in \mathbb{R}^{r}$, assume its desired behaviour is specified by a pair $\{\Psi, \tau\}$, where $\Psi$ is the domain to which the output should be confined and $\tau$ is the period of the confinement, i.e., $y(t) \in \Psi$, for all $t \in[0, \tau]$.

For a given pair $\{\Psi, \tau\}$, the problem of residence time control is formulated as the problem of choosing a feedback control law so as to force the output to remain, at least on the average, in $\Psi$ during period $\tau$, in spite of the disturbances that are acting on the system.

In $[1,2]$ the residence time control problem was analyzed for linear systems with small, additive white noise. It was assumed that all states are available for control and control laws restricted to be linear, state feedback. It has been shown that the class of stabilizable linear systems can be

* This work has been sponsored by the Air Force Office of Scientific Research under Contract F49620-87-C-0079. The U.S. Government's right to retain a nonexclusive royalityfrec license in and to the copyright covering this paper, for governmental purposes, is acknowledged.

${ }^{\dagger}$ Current address: Department of Electrical and Computer Engineering, The Johns Hopkins University, Baltimore, MD 21218. 
divided into two subclasses, weakly and strongly residence time controllable systems. Weakly residence time controllable systems are those systems for which the largest achievable residence time is bounded. On the other hand, strongly residence controllable are those systems for which any residence time is achievable by a choice of $u$.

In the present paper we assume that the control $u$ is based on a measured output that is perturbed by additive noise. We show that due to the measurement noise the largest achievable residence time is always bounded and derive controllers which result in closed loop systems that achieve the maximal residence time.

The structure of the paper is as follows: In Section 2 we briefly review the results of $[1,2]$, in Section 3 we analyze the achievable residence time using direct (static) output feedback, in Section 4 state estimator feedback is considered, in Section 5 we consider an example, and Section 6 is devoted to conclusions. All proofs are given in the Appendix.

\section{Preliminaries and Problem Formulations}

\section{Residence Time Calculation}

Consider the system

$$
\begin{aligned}
d x & =A x d t+\varepsilon C d w, \quad x(0)=x_{0}, \\
y & =D x,
\end{aligned}
$$

where $x \in \mathbb{R}^{n}, y \in \mathbb{R}^{p}, w(t)$ is a standard $r$-dimensional Brownian motion, and $0<\varepsilon \ll 1$ is a parameter.

Let $\Psi$ be a bounded domain in $\mathbb{R}^{p}$ that contains the origin and whose boundary $\partial \Psi$ is smooth. Assume that $x_{0} \in \Omega_{0}=\left\{x \in \mathbb{R}^{p} \mid y=D x \in \Psi\right\}$ and denote the solution of (2.1) with initial condition $x_{n}$ by $y\left(t, x_{0}\right)$. Define the first passage time of $y\left(t, x_{0}\right), x_{0} \in \Omega_{0}$, from $\Psi$ as

$$
\tau^{\varepsilon}\left(x_{0}\right)=\inf \left\{t \geqslant 0 \mid y\left(t, x_{0}\right) \in \partial \Psi\right\} .
$$

Due to the noise in $(2.1), \tau^{\varepsilon}\left(x_{0}\right)$ is a random variable. We denote its mean by $\tau_{\mathrm{ave}}^{\varepsilon}\left(x_{0}\right)$. The following theorem was proven in [2].

Theorem 2.1. Assume that $A$ is Hurwitz, rank $D=p$, and $(A, C)$ is completely disturbable, i.e., $\operatorname{rank}\left[C A C \cdots A^{n-1} C\right]=n$. Then uniformly for all $x_{0}$ belonging to compact subsets of $\Omega=\left\{x \in \mathbb{R}^{n} \mid D e^{A t} x \in \Psi, t \geqslant 0\right\}$ we have

$$
\lim _{\varepsilon \rightarrow 0} \varepsilon^{2} \ln \tau_{\text {ave }}^{\varepsilon}\left(x_{0}\right)=\mu(\Psi)
$$


where

$$
\mu(\Psi)=\min _{y \in \partial \Psi} \frac{1}{2} y^{T}\left(D X D^{T}\right)^{-1} y
$$

and $X$ is the unique positive definite solution of

$$
A X+X A^{T}+C C^{T}=0 .
$$

The constant $\mu(\Psi)$ is referred to as the logarithmic residence time in $\Psi$.

\section{Residence Time Controllability}

Consider now a system with control $u \in \mathbb{R}^{m}$,

$$
\begin{aligned}
d x & =(A x+B u) d t+\varepsilon C d w, \\
y & =D x
\end{aligned}
$$

Assume it is desired to select the control $u=K x$ such that $\tau_{\text {ave }}^{e}\left(K, x_{0}\right) \geqslant T$, where $\tau_{\text {ave }}^{\varepsilon}\left(K, x_{0}\right)$ is the mean first passage time from $\Psi$ of the closed loop system with the indicated control, $T>0$ is some prescribed constant, and $x_{0} \in \Omega_{1}$, where $\Omega_{1}$ is a subset of $\Omega_{0}$ which contains no boundary points of $\Omega_{0}$.

It was shown in $[1\rceil$ that, provided $\varepsilon$ is small enough, the above problem is equivalent to the problem of selecting $K$ such that $\mu(K)>0$, where $\mu(K)$ is given by

$$
\begin{gathered}
\mu(K)=\min _{y \in \dot{\partial} \Psi} \frac{1}{2} y^{T} N(K) y, \\
N(K)=\left(D X(K) D^{T}\right)^{-1}, \\
(A+B K) X(K)+X(K)(A+B K)^{T}+C C^{T}=0 .
\end{gathered}
$$

Definition 2.1. (i) System (2.6) is said to be $y$-wrt controllable if for any bounded $\Psi$, with 0 in its interior, there exists a control $u=K x$ such that $\mu(K)>0$.

(ii) System (2.6) is said to be $y$-srt controllable if for any bounded $\Psi$ $(0 \in \Psi)$ and $\mu>0$ there exists a control $u=K x$ such that $\mu(K) \geqslant \mu$.

In $[1,2]$ conditions for $y$-wrt and $y$-srt controllability were discussed. One of the main results of $[2]$ is the following.

THEOREM 2.2. Assume $(A,[B C])$ is controllable and $(D, A)$ is detectable. Then

(a) (2.6) is $y$-wrt controllable if and only if $(A, B)$ is stabilizable; 
(b) if $(A, B)$ is stabilizable, then (2.6) is $y$-srt controllable if and only if there exists a rational $m \times p$ matrix $U(s)$, with no poles in $\operatorname{Re} s>0$, such that

$$
D(s I-A)^{-1}[B U(s)+C]=0 .
$$

\section{Systems with Noisy Measurements}

In the above discussion attention was restricted to state feedback control laws. This implies, of course, that it is assumed that all states are available for control purposes. In many situations this is not the case, but rather, measured output is available for control. Moreover, in many instances this output is perturbed by additive noise. In the present paper we will assume that this is the case and investigate what effect this restriction has.

We will assume that the measured output is perturbed by small, additive white noise, i.e.,

$$
d z=E z d t+\varepsilon F d w_{1},
$$

where $z, w_{1} \in \mathbb{R}^{q}$ and $0<\varepsilon \ll 1$, and analyze the achievable logarithmic residence time using direct (static) output feedback and state estimator feedback.

In order to avoid trivial situations and to simplify the discussion we make the following assumptions:

A1. $w(t)$ and $w_{1}(t)$ are independent standard Brownian motions and $F F^{T}>0$.

A2. $(A, C)$ is completely disturbable.

A3. $D$ has full rank, i.e., $\operatorname{rank} D=p \leqslant n$.

\section{Direct Output Feedback}

In this section we will discuss the achicvable logarithmic residence time using control laws of the form $u=G d z$ in system (2.6).

The closed loop system with this control is

$$
\begin{aligned}
d x & =(A+B G E) x d t+\varepsilon[C B G F] d w_{0}, \\
y & =D x,
\end{aligned}
$$

where $w_{0}^{T}=\left[w^{T} w_{1}^{T}\right]$. Let $\mathbf{G}=\left\{G \in \mathbb{R}^{m \times q} \mid(A+B G E)\right.$ is Hurwitz $\}$ and define the maximal logarithmic residence time in $\Psi$ as

$$
\mu_{1}^{*}=\sup _{G \in \mathbf{G}} \mu(G),
$$

where $\mu(G)$ is the logarithmic residence time of $(3.1)$. 


\section{An Upper Bound}

In the following theorem we given an upper bound for (3.2).

THEOREM 3.1. Assume that $\mathbf{G}$ is nonempty. Then

$$
\mu_{1}^{*} \leqslant \min _{y \in e^{\Psi}} \frac{1}{2} y^{T}\left(D P D^{T}\right)^{-1} y
$$

where $P$ is the unique positive definite solution of

$$
A P+P A^{T}+C C^{T}-P E^{T}\left(F F^{T}\right)^{-1} E P=0 .
$$

Proof. See the Appendix.

It follows, in particular, from Theorem 3.1 that the logarithmic residence time of any system of the form (3.1) is bounded. Thus, even if (2.6) is $y$-srt controllable, the measurement noise results in a bounded logarithmic residence time.

The following theorem characterizes for which systems the upper bound (3.3) can be attained for some $G \in G$.

THEOREM 3.2. Let $L=-P E^{T}\left(F F^{T}\right)^{-1}$ and let

$$
\mathrm{N}(D, A+B G E)=\bigcap_{i=0}^{n-1} \operatorname{Ker}\left(D(A+B G E)^{i}\right)
$$

be the unobservable subspace of $(D, A \mid B G E)$. Assume there exists $G^{*} \in \mathbf{G}$ such that

$$
\operatorname{Im}\left(B G^{*}-L\right) \subseteq \mathbf{N}\left(D, A+B G^{*} E\right) .
$$

Then the upper bound (3.3) is attained at $G^{*}$.

Conversely, if the upper bound (3.3) is attained at some $G^{*} \in \mathrm{G}$, then there exists a $y^{*} \in \partial \Psi$ such that

$$
\operatorname{Im}\left(B G^{*}-L\right) \subseteq \mathbf{N}\left(\left(y^{*}\right)^{T} D, A+B G^{*} E\right) .
$$

Proof. See the Appendix.

Remark 3.1. It is easy to see from the definition of $\mathbf{N}(D, A+B G E)$ that for $y \in \mathbb{R}^{p}$,

$$
\mathbf{N}(D, A+B G E) \subseteq \mathbf{N}\left(y^{T} D, A+B G E\right) .
$$

Furthermore, if $A+B G E$ is cyclic then equality holds in (3.7) for almost any $y \in \mathbb{R}^{p}[3]$. Thus, for almost any system, (3.5) is a necessary and sufficient condition for the upper bound (3.3) to be attained. 
We will not explore in detail under which conditions on system (2.6) there exists $G^{*} \in \mathbf{G}$ such that (3.5) is satisfied. However, to illustrate the situation, we will consider a special case which is quite often encountered in practice. Assume that the measured output is a linear combination of noisy measurements of the controlled output, i.e.,

$$
d z=R y d t+\varepsilon F d w_{1},
$$

where $R$ is a $q \times p(q \leqslant p)$ matrix. Define

$$
M(D, B)=\left[\begin{array}{cc}
D B \\
D A B \\
\vdots \\
D A^{n} & 1
\end{array}\right] .
$$

Corollary 3.1. Assume $(D, A)$ is detectable and $\operatorname{Im} M(D, B) \supseteq$ $\operatorname{Im} M(D, L)$. Let $G^{*}$ be defined by $M(D, L)=M(D, B) G^{*}$ and assume that $\left(A+B G^{*} E, C\right)$ is disturbable. Then $G^{*} \in \mathbf{G}$ and $\mu\left(G^{*}\right)=\mu_{1}^{*}=$ $\min \left\{\frac{1}{2} y^{T}\left(D P D^{T}\right)^{1} y \mid y \in \partial \Psi\right\}$.

Proof. See the Appendix.

\section{The Optimal Solution}

We now turn to the solution of (3.2) for systems for which Corollary 3.1 does not apply. In order to simplify the discussion, we will concentrate on the special case when the domain $\Psi$ is the ellipsoid $\Psi=\left\{y \in \mathbb{R}^{p}\right\}$ $\left.y^{T} S y \leqslant r^{2}\right\}, S=S^{T}>0, r>0$. Let $W$ be any nonsingular $p \times p$ matrix such that $S=W^{T} W$.

THEOREM 3.3. Assume that for each integer $l \geqslant 1$ the set of equations

$$
\begin{gathered}
\left(A+B G_{l} E\right) X_{l}+X_{l}\left(A+B G_{l} E\right)^{T}+C C^{T}+B G_{l} F F^{T} G_{l}^{T} B^{T}=0 \\
\left(A+B G_{l} E\right)^{T} S_{l}+S_{l}\left(A+B G_{l} E\right)+l D^{T} W^{T}\left(W D X_{l} D^{T} W^{T}\right)^{l} 1{ }^{1} W D \\
G_{l}=-\left(B^{T} S_{l} B\right)^{-1} B^{T} S_{l} X_{l} E^{T}\left(F F^{T}\right)^{-1}
\end{gathered}
$$

has positive definite solutions $X_{l}$ and $S_{l}$. Then $G_{l} \in \mathbf{G}$ and

$$
\lim _{l \rightarrow \infty} \mu\left(G_{l}\right)=\mu_{1}^{*}
$$

Proof. See the Appendix.

The system of equations (3.9)-(3.11) is a system of coupled, nonlinear 
matrix equations and is in general, very difficult to solve. Moreover, it is equally difficult to find sufficient conditions that guarantee the existence of a positive definite solution of (3.9)-(3.11). However, as the following corollary shows, there are special cases in which such conditions can be found.

Corollary 3.2. Assume $\mathbf{G}$ is nonempty, $(D, A)$ is observable, and rank $D=1$. Then for any $l \geqslant 1,(3.9)-(3.11)$ has positive definite solutions $X_{1}$ and $S_{1}$ (independent of $l$ ) and $G_{1}$ is a stabilizing control.

Proof. See the Appendix.

\section{A Suboptimal Solution}

Due to the difficulty of solving (3.9)-(3.11) for $p>1$, it seems advantageous to derive a suboptimal solution to (3.2) for which a solution method exists. One such suboptimal solution can be found using the following considerations. Note that

$$
\begin{aligned}
\mu(G) & =\min _{y \subset \partial \psi} \frac{1}{2} y^{T} N(G) y \\
& \geqslant \frac{1}{2} \lambda_{\min }(N(G)) \min _{y \in \hat{\imath} \Psi} y^{T} y=\frac{\min _{y \in \partial \Psi} y^{T} y}{2 \lambda_{\max }\left(D X(G) D^{T}\right)} \\
& \geqslant \frac{\min _{y \in \partial \Psi} y^{T} y}{2 \operatorname{Tr} D X(G) D^{T}}=v(G) .
\end{aligned}
$$

We will derive a suboptimal solution by maximizing the lower bound $v(G)$ over $G \in \mathbf{G}$.

THEOREM 3.4. Assume $\mathbf{G}$ is nonempty and $(D, A)$ is observable. Then the set of equations

$$
\begin{gathered}
\left(A+B G^{s} E\right) X^{s}+X^{s}\left(A+B G^{s} E\right)^{T}+C C^{T}+B G^{s} F F^{T} G^{s T} B^{T}=0, \\
\left(A+B G^{s} E\right)^{T} S^{s}+S^{s}\left(A+B G^{s} E\right)+D^{T} D=0, \\
G^{s}=-\left(B^{T} S^{s} B\right)^{-1} B^{T} S^{s} X^{s} E^{T}\left(F F^{T}\right)^{-1},
\end{gathered}
$$

has positive definite solutions $X^{s}$ and $S^{s}$ and

$$
\mu\left(G^{s}\right)=\max _{G \in \mathbf{G}} v(G) .
$$

Proof. See the Appendix.

Numerical methods for solving equations of the form (3.14)-(3.16) have 
been discussed in $[4,5]$. Thus, Theorem 3.4 gives a computable suboptimal solution to (3.2).

\section{Relationship with Optimal Output Control}

The optimization problem (3.2), i.e., $\max \{\mu(G) \mid G \in \mathbf{G}\}$ subject to

$$
(A+B G E) X(G)+X(G)(A+B G E)^{T}+C C^{T}+B G F F^{T} G^{T} B^{T}=0,
$$

is closely related to the optimal output control problem introduced in [6] and further analyzed in [5-7]. Indeed, in the optimal output control problem one considers the deterministic system

$$
\begin{aligned}
& \dot{x}=A_{1} x+B_{1} u, \quad x(0)=x_{0}, \\
& y=D_{1} x,
\end{aligned}
$$

and seeks an output control law $u=K_{1} y$ so as to minimize the cost

$$
J(u)=\int_{0}^{\alpha}\left(x^{T}(t) Q x(t)+u^{T}(t) R u(t)\right) d t,
$$

where $Q \geqslant 0$ and $R>0$.

It is well known that (3.19) can be written as

$$
J\left(K_{1}\right)=x_{0}^{T} S\left(K_{1}\right) x_{0},
$$

where

$$
\left(A_{1}+B_{1} K_{1} D_{1}\right)^{T} S\left(K_{1}\right)+S\left(K_{1}\right)\left(A_{1}+B_{1} K_{1} D_{1}\right)+Q+D_{1}^{T} K_{1}^{T} R K_{1} D_{1}=0 .
$$

The similarity between (3.17) and (3.21) is now apparent. Indecd, if let $A_{1}=A^{T}, \quad D_{1}=B^{T}, \quad B_{1}=E^{T}, \quad Q=C C^{T}, R=F F^{T}$, and $K_{1}=G^{T}$, then $S\left(K_{1}\right)=X(G)$. Thus, if (3.18) and (3.19) are defined in the dual fashion described above, then (3.2) and the optimal output control problem are related in the sense that both involve the optimization of a quadratic functional in $X(G)$.

It can be shown that the optimal solution (if it exists) to the optimal output control problem depends on the initial point $x_{0}$. However, in general $x_{0}$ is unknown and, therefore, the optimal control is not completely defined. To eliminated this difficulty suboptimal solutions have been proposed. In [5-7] a suboptimal solution was constructed by assuming that $x_{0}$ is a random variable with covariance $X_{0}=E\left[x_{0} x_{0}^{T}\right]$ and a new cost index was defined as $J_{\text {ave }}\left(K_{1}\right)=E\left[J\left(K_{1}\right)\right]=\operatorname{Tr} S\left(K_{1}\right) X_{0}$. In [8] the 
dependence on $x_{0}$ was eliminated by considering the worst case cost $J_{\text {wor }}\left(K_{1}\right)=\sup \left\{x_{0}^{T} S\left(K_{1}\right) x_{0} \mid x_{0}^{T} x_{0}=1\right\}$.

The suboptimal solution discussed in [8], i.e., $\min \left\{J_{\text {wor }}\left(K_{1}\right) \mid K_{1}^{\prime} \in \mathbf{G}\right\}$ subject to (3.21), is exactly the dual of the optimal solution to (3.2) discussed previously in this section, i.e., if $W=I$ and (3.18) and (3.19) are defined as before, then the optimal $K_{1}$ which minimizes $J_{\text {wor }}\left(K_{1}\right)$ is equal to the transpose of the optimal solution described in Theorem 3.3. Furthermore, if we choose $X_{0}=D D^{T}$ then the suboptimal solution of the optimal output control problem discussed in [5-7] is the dual of the suboptimal solution to (3.2) discussed previously in this section.

\section{State Estimator Feedback}

In this section we assupe that the control law $u$ is based on an estimate of the state $x$ and study the achievable logarithmic residence time using control laws of this form. In particular, let the estimate $x_{\mathrm{e}}$ of $x$ be generated by the estimator

$$
d x_{\mathrm{e}}=\left(A x_{\mathrm{e}}+B u\right)+L\left(d z-E x_{\mathrm{e}} d t\right)
$$

and consider control laws of the form

$$
u=K x_{\mathrm{e}} .
$$

Define

$$
\begin{aligned}
& \mathbf{K}=\left\{K \in \mathbb{R}^{m \times n} \mid(A+B K) \text { is Hurwitz }\right\}, \\
& \mathbf{L}=\left\{L \in \mathbb{R}^{n \times q} \mid(A-L E) \text { is Hurwitz }\right\}
\end{aligned}
$$

and let $\mu(K, L)$ be the logarithmic residence time in $\Psi$ of the closed loop systcm with control $u=K x_{\mathbf{c}}, K \in \mathbf{K}, L \in \mathbf{L}$. Definc the maximal logarithmic residence time in $\Psi$ as

$$
\mu_{2}^{*}=\max _{K \in \mathbf{K}, L \in \mathbf{L}} \mu(K, L)
$$

\section{An Upper bound}

Theorem 4.1. Assume that $(A, B)$ is stabilizable and $(E, A)$ is detectable. Then

$$
\mu_{2}^{*} \leqslant \min _{y \in \partial \Psi} \frac{1}{2} y^{T}\left(D P D^{T}\right)^{-1} y
$$

where $P$ is the unique positive definite solution of (3.4). 
Proof. See the Appendix.

The upper bound (4.4) is the same as the bound in (3.3). Therefore, for systems for which the bound ( 3.3 ) can be attained by a choice of controller $G \in \mathbf{G}$, nothing is gained by using the more complex controller $(4.1)-(4.2)$. Note, however, that typically the class of control laws $(\mathbf{K}, \mathbf{L})$ is much larger than the class $\mathbf{G}$.

Define

$$
\begin{gathered}
G_{s}(s)=D(s I-A)^{-1} B, \\
G_{l}(s)=D(s I-A)^{\prime}{ }^{\prime} L^{*}, \quad L^{*}=P E^{T}\left(F F^{T}\right) \quad
\end{gathered}
$$

The following theorem gives conditions under which the upper bound (4.4) is attained.

Theorfm 4.2. Assume $(A, B)$ is stabilizable and $(D, A)$ and $(E, A)$ are detectable. Then the upper bound (4.4) is attained if and only if there exists a rational $m \times q$ matrix $W(s)$, with no poles in $\operatorname{Re} s>0$, such that

$$
G_{l}(s)+G_{s}(s) W(s)=0 .
$$

Proof. See the Appendix.

In order to compare Theorem 4.2 with Theorem 3.2, which gives the analogous conditions for direct output feedback control laws, we state the following corollary.

Corollary 4.1. Assume that there exists a $G \in \mathbb{R}^{m \times 4}$ such that

$$
\operatorname{Im}\left(B G-L^{*}\right) \subseteq \mathbf{N}(D, A) .
$$

Then the upper bound is attained.

Proof. See the Appendix.

It follows, in particular, from Corollary 4.1 that the class of systems for which the upper bound (3.3) is attained belongs to the class of systems for which (4.4) is attained.

\section{The Optimal Solution}

What remains to be solved is to evaluate the maximal logarithmic residence time $\mu_{2}^{*}$. In order to simplify the discussion, we assume in the remainder of the section that $y$ is a scalar. 
Theorem 4.3. Assume $(A, B)$ is stabilizable, $(D, A)$ and $(E, A)$ are detectable, and $\Psi=(-a, b), a, b>0$. Then

$$
\mu_{2}^{*}=\frac{(\min (a, b))^{2}}{2 \operatorname{Tr}\left(C^{T} Q_{0} C+K_{0} P K_{0}^{T}\right)},
$$

where $P$ is given by (3.4) and

$$
\begin{gathered}
Q_{0}=\lim _{\gamma \rightarrow 0} Q_{\gamma}, \\
K_{0}=\lim _{\gamma \rightarrow 0} \frac{1}{\sqrt{\gamma}} B^{T} Q_{\gamma}, \\
A^{T} Q_{\gamma}+Q_{\gamma} A+D^{T} D-\frac{1}{\gamma} Q_{\gamma} B B^{T} Q_{\gamma}=0 .
\end{gathered}
$$

Proof. See the Appendix.

\section{EXAMPLE}

Consider the second-order system

$$
\left[\begin{array}{l}
d x_{1} \\
d x_{2}
\end{array}\right]=\left[\begin{array}{rr}
0 & 1 \\
-1 & 0
\end{array}\right]\left[\begin{array}{l}
x_{1} \\
x_{2}
\end{array}\right] d t+\left[\begin{array}{l}
0 \\
1
\end{array}\right](u d t+\varepsilon d w)
$$

Let $d z=E x d t+F d w_{1}$ be a measured output and assume it is desired to select the control $u=u(d z)$ such that the residence time of $y(t)=x_{1}(t)$ in the interval $(-1,1)$ is maximized. Note that the system transfer function for $(5.1)$ is

$$
G_{s}(s)=D(s I-A)^{-1} B=\frac{1}{s^{2}+1} .
$$

Therefore, since $G_{s}(s)$ has no finite zeroes, it follows from Theorem 4.2 that

$$
\mu_{2}^{*}=\frac{1}{2 D P D^{T}}
$$

Below we consider three cases of a measured output $d z$ and compare the achievable logarithmic residence time using the control laws discussed in the previous sections.

1. $d z=x_{1} d t+\varepsilon d w_{1}$. In this case $E=\left[\begin{array}{ll}1 & 0\end{array}\right]$ and it is easy to see that 
$\mathbf{G}$ is empty. On the other hand, since $(A, B)$ is controllable and $(E, A)$ is observable, the control

$$
\begin{aligned}
& L^{*}=P E^{T}\left(F F^{T}\right)^{-1}=\left[\begin{array}{r}
0.901 \\
-0.414
\end{array}\right], \\
& K^{\gamma}=-\left[\sqrt{1+\frac{1}{\gamma}}-1 \sqrt{2} \sqrt{1+\sqrt{1+\frac{1}{\gamma}}}\right],
\end{aligned}
$$

results in a logarithmic residence time, $\mu\left(K^{\prime}, L^{*}\right)$, which converges in the limit $\gamma \rightarrow 0$ to

$$
\mu_{2}^{*}=0.549 .
$$

2. $d z=x_{2} d t+\varepsilon d w_{1}$. In this case $E=\left[\begin{array}{ll}0 & 1\end{array}\right]$ and it $\mathbf{G}$ is nonempty. Furthermore, for any $G$, the pair $(D, A+B G E)$ is observable. Thus, by Theorem 3.2, the upper bound (3.3) is attained if there exists a $G^{*}$ such that $B G^{*}-L=0$. A simple calculation shows that $L=-\left[\begin{array}{ll}0 & 1\end{array}\right]^{T}$. Therefore, the control law $u=G^{*} z=-z$ results in a logarithmic residence time

$$
\mu(-1)=\mu_{1}^{*}=0.5 \text {. }
$$

Note that in order to achieve the logarithmic residence time (5.7) using a state estimator feedback requires infinite control gains. Thus, in this case, the advantage of the direct output feedback is considerable.

3. $d z=I x d t+\varepsilon I d w_{1}(I=2 \times 2$ identity $)$. Obviously, $\mathbf{G}$ is nonempty. The pair $(D, A+B G E)$ is observable for any $G$. Thus, the upper bound (3.3) is attained for some $G^{*} \in \mathbf{G}$ if and only if $B G^{*}-L=0$. However, it is easy to see that $L=-P$; thus, there exists no $G^{*}$ such that $(3.3)$ is attained. Solving (3.9)-(3.11) for the optimal control gives $G_{1}=$ $-\left[\begin{array}{ll}1 & 0.707\end{array}\right]$. The logarithmic residence time with this control is

$$
\mu\left(G_{1}\right)=\mu_{1}^{*}=0.707 .
$$

Solving (3.4) for $P$ gives

$$
P=\left[\begin{array}{rr}
0.560 & -0.172 \\
-0.172 & 0.792
\end{array}\right]
$$

and the upper bound (3.3), (4.4) is

$$
\mu_{2}^{*}=0.893 .
$$

Note that the largest achievable logarithmic residence time $\mu_{2}^{*}$ is only $25 \%$ improvement over $\mu_{1}^{*}$. However, in order to obtain a logarithmic residence time which is larger than $\mu_{1}^{*}$ requires a large control gain. 


\section{CONCLUSION}

In this paper we considered the problem of residence time control for linear systems perturbed by small input and measurement noise. It was shown that, due to the measurement noise, the maximal residence time is always bounded. This phenomenon can be explained as being result of the amplification of the measurement noise by the control "gain."

In conclusion, even if a system is $y$-srt controllable, i.e., any residence time is achievable by a state feedback control law, any amount of measurement noise will result in a bounded residence time. Therefore, measurement noise has greater limiting effect than input noise on the achievable residence time.

\section{APPENDIX}

Proof of Theorem 3.1. The logarithmic residence time of (3.1) is given by (for $G \in \mathbf{G}$ )

$$
\begin{aligned}
(A+B G E) X(G)+X(G)(A+B G E)^{T}+C C^{T}+B G F F^{T} G^{T} B^{T}=0, \\
N(G)=\left(D X(G) D^{T}\right)^{-1}, \\
\mu(G)=\min _{y \in \partial \psi} \frac{1}{2} y^{T} N(G) y .
\end{aligned}
$$

Note that (3.4) can be rewritten as

$$
\begin{aligned}
(A+ & B G E) P+P(A+B G E)^{T}+C C^{T} \\
& -P E^{T}\left(F F^{T}\right)^{-1} E P-B G E P-P E^{T} G^{T} B^{T}=0 .
\end{aligned}
$$

Subtracting (A.4) from (A.1) gives

$$
\begin{aligned}
(A+ & B G E)(X(G)-P)+(X(G)-P)(A+B G E)^{T}+B G F F^{T} G^{T} B^{T} \\
& +P E^{T}\left(F F^{T}\right)^{-1} E P+B G E P+P E^{T} G^{T} B^{T}=0
\end{aligned}
$$

The last four terms in (A.5) can be rewritten as

$$
W=\left(B G+P E^{T}\left(F F^{T}\right)^{-1}\right) F F^{T}\left(B G+P E^{T}\left(F F^{T}\right)^{-1}\right)^{T} .
$$

Obviously, $W \geqslant 0$. Therefore, since $(A+B G E)$ is Hurwitz, it follows from a standard Liapunov theorem that $X(G)-P \geqslant 0$. This implies that $D X(G) D^{T} \geqslant D P D^{T}$ and since $(A, C)$ is disturbable we have $P>0$ and thus $D P D^{T}>0$. Therefore, $\left(D P D^{T}\right)^{-1} \geqslant\left(D X(G) D^{T}\right)^{-1}$ and (3.3) follows.

Q.E.D. 
Proof of Theorem 3.2. First note that (A.6) can be written as

$$
W=(B G-L) F F^{T}(B G-L)^{T} .
$$

From (A.5) and (A.7) it follows that for any $G \in \mathbf{G}$

$$
\begin{aligned}
& D(X(G)-P) D^{T} \\
& \quad=\int_{0}^{\infty} D e^{(A+B G E) \iota}(B G-L) F F^{T}(B G-L)^{T} e^{(A+B G E)^{T} t} D^{T} d t .
\end{aligned}
$$

Assume now that (3.5) is satisfied for $G^{*} \in \mathbf{G}$. Then it is easy to show that

$$
D\left(A+B G^{*} E\right)^{i}\left(B G^{*}-L\right)=0, \quad i=0,1, \ldots, n-1,
$$

and, in particular,

$$
D e^{\left(A+B G^{*} E\right) t}\left(B G^{*}-L\right)=0, \quad t \geqslant 0 .
$$

Whence, the integrand in (A.8) is identically zero and, therefore, $D X(G) D^{T}=D P D^{T}$. This completes the proof of the first part.

To prove the second part of the theorem assume that $(3.3)$ is attained at some $G^{*} \in \mathbf{G}$. Then there exist points $y_{1}$ and $y_{2}$ belonging to $\partial \Psi$ such that

$$
\begin{aligned}
\min _{y \in \grave{c} \Psi} y^{T}\left(D X\left(G^{*}\right) D^{T}\right)^{-1} y & =y_{1}^{T}\left(D X\left(G^{*}\right) D^{T}\right)^{-1} y_{1} \\
& =y_{2}^{T}\left(D P D^{T}\right)^{-1} y_{2} \\
& =\min _{y \in \partial \Psi} y^{T}\left(D P D^{T}\right)^{-1} y .
\end{aligned}
$$

Furthermore,

$$
y_{1}^{T}\left(D X\left(G^{*}\right) D^{T}\right)^{-1} y_{1} \leqslant y^{T}\left(D X\left(G^{*}\right) D^{T}\right)^{-1} y
$$

for all $y \in \partial \Psi$. Thus, by (A.9)-(A.10),

$$
y_{2}^{T}\left(D X\left(G^{*}\right) D^{T}\right)^{-1} y_{2} \geqslant y_{2}^{T}\left(D P D^{T}\right)^{-1} y_{2} .
$$

From the proof of Theorem 3.1 we know that

$$
\left[\left(D P D^{T}\right)^{-1}-\left(D X\left(G^{*}\right) D^{T}\right)^{-1}\right] \geqslant 0 .
$$

From (A.11) and (A.12) we conclude that

$$
y_{2}^{T}\left(D X\left(G^{*}\right) D^{T}\right)^{-1} y_{2}=y_{2}^{T}\left(D P D^{T}\right)^{-1} y_{2} .
$$


Next we will show that there exists a point $y^{*}$ such that

$$
y^{* T}\left[D P D^{T}-D X\left(G^{*}\right) D^{T}\right] y^{*}=0 .
$$

The matrix $\left(D P D^{T}\right)^{-1}$ is positive definite. Thus, there exists a nonsingular $Q \in \mathbb{R}^{p \times p}$ such that $\left(D P D^{T}\right)^{-1}=Q^{T} Q$. From (A.12) wc get

$$
Q^{T}\left[I-Q^{-T}\left(D X\left(G^{*}\right) D^{T}\right)^{-1} Q^{-1}\right] Q \geqslant 0 .
$$

The matrix $Q^{-T}\left(D X\left(G^{*}\right) D^{T}\right)^{-1} Q^{-1}$ is positive definite. Thus, there exists a unitary matrix $U$ which diagonalizes it, i.e.,

$$
Q^{-T}\left(D X\left(G^{*}\right) D^{T}\right)^{-1} Q^{-1}=U^{T} S U
$$

where $S=\operatorname{diag}\left(s_{1}, s_{2}, \ldots, s_{p}\right)$. Equations (A.15) and (A.16) give

$$
Q^{T} U^{T}[I-S] U Q \geqslant 0 .
$$

Thus, $s_{i} \leqslant 1, i=1, \ldots, p$. Let $z_{2}=U Q y_{2}$, where $y_{2}$ is given by (A.9), (A.13). Then it follows from (A.13) and (A.17) that

$$
z_{2}^{T}[I-S] z_{2}=0
$$

Therefore, for each $i$ we either have $\left(1-s_{i}\right)=0$ or $z_{2}^{i}=0, i=1, \ldots, p$. We know that for some $j, z_{2}^{j} \neq 0$ (since $y_{2} \neq 0$ and $U Q$ is nonsingular). Next note that $D P D^{T}=Q^{-1} Q^{-T}$. Therefore,

$$
\begin{aligned}
D P D^{T}-D X\left(G^{*}\right) D^{T} & =Q^{-1} Q^{-T}-D X\left(G^{*}\right) D^{T} \\
& =Q^{-1}\left[I-Q D X\left(G^{*}\right) D^{T} Q^{T}\right] Q^{-T} \\
& =Q^{-1}\left[I-\left(Q^{-T}\left(D X\left(G^{*}\right) D^{T}\right)^{-1} Q^{-1}\right)^{-1}\right] Q^{-T} \\
& =Q^{-1}\left[I-\left(U^{T} S U\right)^{-1}\right] Q^{-T} \\
& =Q^{-1} U^{-1}\left[I-S^{-1}\right] U^{-T} Q^{-T}
\end{aligned}
$$

We want to find $y^{*}$ so that (A.14) is satisfied. Equivalently, we want to find $z^{*}$ such that $z^{* T}\left[I-S^{-1}\right] z^{*}=0$. Note that $z_{2}^{T}\left[I-S^{-1}\right] z_{2}=0$. Define $y^{*}$ by $z_{2}=U^{-T} Q^{-T} y^{*}$. Then $y^{*}=Q^{T} U^{T} z_{2}=Q^{T} U^{T} U Q y_{2}=Q^{T} Q y_{2}=$ $\left(D P D^{T}\right)^{-1} y_{2}$ satisfies (A.14). The equality (A.14) implies that (by (A.8))

$$
0=\int_{0}^{\infty} y^{* T} D e^{\left(A+B G^{*} E\right) t}\left(B G^{*}-L\right) F F^{T}\left(B G^{*}-L\right)^{T} e^{\left(A+B G^{*} E\right)^{T} t} D^{T} y^{*} d t
$$

The integrand in (A.20) is nonnegative; therefore, the integral can be zero 
if and only if the integrand is identically zero. Furthermore, since $F F^{I}$ is positive definite this implies that

$$
y^{* T} D e^{\left(A+B G^{*} E\right) !}\left(B G^{*}-L\right)=0,
$$

which in turn implies (3.6).

Q.E.D.

Proof of Corollary 3.1. It is not difficult to show that if $M(D, L)=$ $M(D, B) G^{*}$, then $\mathbf{N}(D, A) \supseteq \operatorname{Im}\left(B G^{*}-L\right)$. Furthermore, since $E=R D$, it follows from the definition of $\mathbf{N}(D, A)$ that $\mathbf{N}(D, A)=\mathbf{N}\left(D, A+B G^{*} E\right)$. Therefore, under the stated assumptions,

$$
\operatorname{Im}\left(B G^{*}-L\right) \subseteq \mathbf{N}\left(D, A+B G^{*} E\right),
$$

and by Theorem 3.2, if $G^{*} \in \mathbf{G}$, then the upper bound (3.3) is attained at $G^{*}$. Thus, the proof is complete if we can show that any $G^{*}$ which satisfies (A.22) belongs to $\mathbf{G}$.

From (A.5), (A.7), and [9, pp. 603] there follows that for $0 \leqslant t<\infty$

$$
\begin{aligned}
D\left[X\left(G^{*}\right)-P\right] D^{T}= & D e^{\left(A+B G^{*} E\right) t}\left[X\left(G^{*}\right)-P\right] e^{\left(A+B G^{*} E\right)^{T} t} D^{T} \\
& +\int_{0}^{t} D e^{\left(A+B G^{*} E\right) s} W e^{\left(A+B G^{*} E\right)^{T_{s}}} D^{T} d s .
\end{aligned}
$$

From (A.22) it follows that the integral term in (A.23) is identically zero. Thus, for any $0 \leqslant t<\infty$ we have

$$
D\left[X\left(G^{*}\right)-P\right] D^{T}=D e^{\left(A+B G^{*} E\right)^{4}}\left[X\left(G^{*}\right)-P\right] e^{\left(A+B G^{*} E\right)^{\prime} t} D^{T} .
$$

Equation (A.24) can be true only if both sides are identically zero. Therefore, $D X\left(G^{*}\right) D^{T}=D P D^{T}>0$, and since $(D, A)$ is detectable and $\left(A+B G^{*} E, C\right)$ is disturbable it follows from [10, Corollary 1] that $G^{*} \in \mathbf{G}$.

Q.E.D.

Proof of Theorem 3.3. When $\Psi$ is an ellipsoid the logarithmic residence time can be written as

$$
\begin{aligned}
\mu(G) & =\min _{y^{T} S y=r^{2}} \frac{1}{2} y^{T} N(G) y \\
& =\min _{z^{T} z=r^{2}} \frac{1}{2} z^{T} W^{-T} N(G) W^{-1} z \\
& =\min _{z^{T} z=r^{2}} \frac{1}{2} z^{T}\left(W D X(G) D^{T} W^{T}\right)^{-1} z \\
& =\frac{1}{2} \lambda_{\min }\left(\left(W D X(G) D^{T} W^{T}\right)^{-1}\right) r^{2} \\
& =\frac{r^{2}}{2 \lambda_{\max }\left(W D X(G) D^{T} W^{T}\right)} .
\end{aligned}
$$


It follows from (A.25) that (3.2) is equivalent to the minimization problem

$$
\min _{G \in \mathbf{G}} \lambda_{\max }\left(W D X(G) D^{T} W^{T}\right) .
$$

It can be proven that $[8]$

$\min _{G \in \mathbf{G}} \lambda_{\max }\left(W D X(G) D^{T} W^{T}\right)=\lim _{l \rightarrow \infty}\left(\min _{G \in \mathbf{G}} \operatorname{Tr}\left(W D X(G) D^{T} W^{T}\right)^{l}\right)^{1 / 2}$.

It is straightforward to show (using, e.g., the techniques of [11]) that (3.9)-(3.11) is a necessary condition for $G_{l}$ to minimize $\operatorname{Tr}(W D X(G)$ $\left.D^{T} W^{T}\right)^{l}$. Next, it follows from a standard Liapunov type theorem [12] that the positive definiteness of $X_{I}$ and the disturbability of $(A, C)$ imply that $G_{l} \in$ G. Finally, (3.12) follows from (A.27).

Q.E.D.

Proof of Corollary 3.2. In the case $p=1$, Eq. (3.10) becomes

$$
\left(A+B G_{l} E\right)^{T} S_{l}+S_{l}\left(A+B G_{l} E\right)+l W^{2}\left(W D X_{l} D^{T} W\right)^{l-1} D^{T} D=0 .
$$

Thus,

$$
S_{l}=l\left(W D X_{l} D^{T} W\right)^{l-1} S^{*}
$$

where $S^{*}$ satisfies

$$
\left(A+B G_{l} E\right)^{T} S^{*}+S^{*}\left(A+B G_{l} E\right)+W^{2} D^{T} D=0
$$

and (3.11) becomes

$$
G_{l}=-\left(B^{T} S^{*} B\right)^{-1} B^{T} S^{*} X_{l} E^{T}\left(F F^{T}\right)^{-1} .
$$

Note that the system of equations (3.9), (A.30), and (A.31) is independent of $l$ (this is easily seen by substituting (A.31) into (3.9)). Finally, it follows from the results of [7] and the observability of $(D, A)$ that the system (3.9), (A.30), and (A.31) has positive definite solutions $X_{1}$ and $S_{1}$ and $G_{1}$ is a stabilizing control.

Q.E.D.

Proof of Theorem 3.4. The proof of this theorem follows directly from the results of [7] and the observability of $(D, A)$ Q.E.D.

Proof of Theorem 4.1. The logarithmic residence time of the closed loop system with control (4.1)-(4.2) is $(K \in \mathbf{K}, L \in \mathbf{L})$

$$
\mu(K, L)=\min _{y \in i \Psi} y^{T}\left(D X(K, L) D^{T}\right)^{-1} y,
$$


where

$$
\begin{aligned}
{\left[\begin{array}{cc}
A & B K \\
L E & A+B K-L E
\end{array}\right]\left[\begin{array}{cc}
X(K, L) & T(K, L) \\
T^{T}(K, L) & X_{\mathrm{e}}(K, L)
\end{array}\right] } \\
+\left[\begin{array}{cc}
X(K, L) & T(K, L) \\
T^{T}(K, L) & X_{\mathrm{e}}(K, L)
\end{array}\right]\left[\begin{array}{cc}
A & B K \\
L E & A+B K-L E
\end{array}\right] \\
+\left[\begin{array}{cc}
C C^{T} & 0 \\
0 & L F F^{T} L^{T}
\end{array}\right]=0 .
\end{aligned}
$$

It is straightforward to show (e.g., [13]) that for any $K \in \mathbf{K}$ and $L \in \mathbf{L}$, $X(K, L) \geqslant P$, where $P$ is given by (3.4). Furthermore, under the stated assumptions $P>0$ and, thus, (4.4) follows.

Q.E.D.

Proof of Theorem 4.2. In a system with the estimator gain $L^{*}$ and any control gain $K \in \mathbf{K}$ we have

$$
X\left(K, L^{*}\right)=X_{\mathrm{e}}\left(K, L^{*}\right)+P,
$$

where $X_{e}\left(K, L^{*}\right)$ is the positive definite solution of

$$
(A+B K) X_{\mathrm{e}}\left(K, L^{*}\right)+X_{\mathrm{e}}\left(K, L^{*}\right)(A+B K)^{T}+L L^{* T}=0 .
$$

It follows from (A.34) that the upper bound (4.4) is attained if and only if

$$
\inf _{K \in \mathbf{K}} \operatorname{Tr} X_{\mathrm{e}}\left(K, L^{*}\right)=0 .
$$

Finally, it was shown in [2] that (4.5) is a necessary and sufficient condition for (A.36) to be satisfied.

Q.E.D.

Proof of Corollary 4.1. If (4.6) is true then

$$
D A^{i}\left(B G-L^{*}\right)=0, \quad i=0,1, \ldots, n-1,
$$

and, in particular,

$$
D(s I-A)^{-1}\left(B G-L^{*}\right)=0 .
$$

It follows from (A.38) that (4.5) is satisfied with $W(s)=G=$ const. Q.E.D.

Proof of Theorem 4.3. In the scalar case the logarithmic residence time in the interval $\Psi=(-a, b), a, b>0$, is

$$
\mu(K, L)=\frac{(\min (a, b))^{2}}{2 D X(K, L) D^{T}} .
$$


It is easy to see that the supremum over $K$ in (4.3) is not attained at any $K \in \mathbf{K}$ (due to the fact that (A.33) is linear in $K$ ). To overcome any difficulty we regularize (A.39) in $K$. Define a regularized "cost" as

$$
\mu_{i}(K, L)=\frac{(\min (a, b))^{2}}{2\left(D X(K, L) D^{T}+\gamma \operatorname{Tr} K X_{\mathrm{e}}(K, L) K^{T}\right)} .
$$

We will maximize $\mu_{\gamma}(K, L)$ over $K \in \mathbf{K}$ and $L \in \mathbf{L}$ and then take the limit $\gamma \rightarrow 0$ and show that the optimum value of $\mu_{\gamma}(K, L)$ converges to $\mu_{2}^{*}$.

Note that maximizing $\mu_{\gamma}(K, L)$ is equivalent to minimizing

$$
J_{y}(K, L)=D X(K, L) D^{T}+\gamma \operatorname{Tr} K X_{\mathrm{e}}(K, L) K^{T} .
$$

It is wcll known from the theory of optimal control (c.g., [11]) that $J_{\gamma}(K, L)$ is minimized by

$$
\begin{array}{ll}
K^{\gamma}=-\frac{1}{\gamma} B^{T} Q_{\gamma}, & A^{T} Q_{\gamma}+Q_{\gamma} A+D^{T} D-\frac{1}{\gamma} Q_{\gamma} B B^{T} Q_{\gamma}=0, \\
L^{*}=P E^{T}\left(F F^{T}\right)^{-1}, & A P+P A^{T}+C C^{T}-P E^{T}\left(F F^{T}\right)^{-1} E P=0 .
\end{array}
$$

Furthermore, it can be shown that [13]

$$
J_{\gamma}\left(K^{\prime}, L^{*}\right)=\operatorname{Tr}\left(C^{T} Q_{\gamma} C+\gamma K^{\prime} P K^{\prime T}\right) .
$$

As $\gamma \rightarrow 0, J_{\gamma}\left(K^{\gamma}, I^{*}\right)$ converges to $C^{T} Q_{0} C+K_{0} P K_{0}^{T}$ (the existence of the limits is proven in [14]).

What remains to be shown is that $\gamma \operatorname{Tr}\left(K^{\gamma} X_{\mathrm{e}}\left(K^{\gamma}, L^{*}\right) K^{\gamma T}\right)$ converges to zero as $\gamma \rightarrow 0$. The proof of this is a minor modification of the main theorem in [14] and is omitted.

Q.E.D.

\section{REFERENCES}

1. S. M. Meerkov an T. Runolfsson, Residence time control, IEEE Trans. Automat. Contr. AC-33, No. 4 (1988).

2. S. M. MeErkov and T. Runolfsson, Output residence time control, IEEE Trans. Automat. Contr. AC-34, No. 11 (1989).

3. C-T. ChEN, "Linear System Theory and Design," Holt, Rinehart \& Wintson, New York, 1984.

4. B. D. O. ANDerson AND J. B. MoORe, "Linear Optimal Control," Prentice-Hall, Englewood Cliffs, NJ, 1971.

5. H. P. Horisberger and P. T. Belanger, Solution of the constant output feedback problem by conjugate gradients, IEEE Trans. Automal. Contr. AC-19 (1974).

6. W. S. LeVINE AND M. ATHANS, On the determination of the optimal constant output feedback gains for linear multivariable systems, IEEE Trans. Automat. Contr. AC-15 (1970). 
7. J. Medanic, On stabilization and optimization by output feedback, in "Proceedings, Asilomar Conference on Circuits, Systems and Computers, Pacific Grove, CA, 1979."

8. J. C. Allwright ani) J. Q. Mao, Optimal output feedback by minimizing $\|K(F)\|_{2}$, IEEE Trans. Automat. Contr. AC-27 (1982).

9. T. Kallath, "Linear Systems," Prentice-Hall, Englewood Cliffs, NJ, 1980.

10. J. SNYDERS, Stationary probability distributions for linear time-invariant systems, SIAM J. Control Optim. 15 (1977).

11. D. L. Russell, "Mathematics of Finite-Dimensional Control Systems," Dekker, New York, 1979.

12. W. M. Wonham, "Linear Multivariable Control," 3rd ed., Springer-Verlag, New York, 1985.

13. A. E. Bryson, JR., AND Y.C. Ho, “Applied Optimal Control," Hemisphere Publishing, Washington, DC, 1975.

14. H. KWAKERnaAk aNd R. Sivan, The maximally achievable accuracy of linear optimal regulators and linear optimal filters, IEEE Trans. Automat. Contr. AC-17 (1972). 